\title{
ORAL CORNIFICATION IN ANAEMIC PATIENTS
}

\author{
BY \\ ALLAN JACOBS* \\ From the Department of Clinical Pathology, Charing Cross Hospital Medical School, London
}

(RECEIVED FOR PUBLICATION JUNE 21, 1958)

Changes in the oral epithelium in irondeficiency anaemia have long been recognized, and the clinical picture of glossitis and stomatitis, sometimes in association with dysphagia, is especially familiar in women with "idiopathic" hypochromic anaemia (Paterson, 1919). Waldenström (1938) has shown that these changes may occur in cases of occult iron deficiency in which anaemia has not yet developed. Despite wide appreciation of the oral symptoms little investigation of the epithelium itself in this condition has been carried out.

The normal buccal epithelium has been previously investigated by means of smears fixed and stained by Papanicolaou's technique (Jacobs, 1958). The present investigation is concerned with the buccal cornification in patients with anaemia due to iron deficiency and other causes.

\section{A Survey of Anaemic Patients}

Three groups were examined.

(1) Thirty-nine cases (18 male, 21 female) of iron-deficiency anaemia due to gastro-intestinal haemorrhage caused by haemorrhage from duodenal ulcer 13, carcinoma of large intestine nine, post-gastrectomy bleeding six, hiatus hernia five, gastric ulcer three, gastric carcinoma three. deficiency anaemia in women all of which presented with symptoms of anaemia without any evidence of haemorrhage. In some cases there was a complaint of menorrhagia.

\footnotetext{
*Present address: Department of Pathology, Paddington General Hospital, Harrow Road, W.2.
}

(2) Twenty cases of "idiopathic" iron-

(3) Twenty-five cases (12 male, 13 female) of anaemia without iron deficiency, caused by pernicious anaemia seven, chronic lymphatic leukaemia six, chronic myeloid leukaemia four, myelomatosis six, and acute leukaemia two. The degree of anaemia in these cases is shown in the Table.

Papanicolaou-stained smears from the inside of the cheek were examined and the percentage of squamous cells showing cytoplasmic acidophilia or orangeophilia (cornification index) was calculated in each case.

\section{Results}

The cornification indices in the three anaemic groups are shown in Fig. 1 where they are compared with the findings in 200 normal subjects (Jacobs, 1959). The mean cornification indices of these groups are:

$$
\begin{array}{lrrr}
\text { Normal } \quad . \quad . & . & 36.1 \pm 1.4 \\
\text { Group } 1 \text { (post-haemorrhagic) } & 33.3 \pm 2.7 \\
\begin{array}{l}
\text { Group } 2 \text { ("idiopathic ") } \\
\text { Group 3 (leukaemias }
\end{array} & \text { and } & 71.9 \pm 3.6 \\
\text { pernicious anaemia) } & . & 38.4 \pm 3.8
\end{array}
$$

The anaemic patients in Groups 1 and 3 do not vary significantly from the normal, but the increase in the cornification index in Group 2 is highly significant $(P<0.0001)$. In none of the groups is there any correlation between cornification and the haemoglobin level.

\section{The Response of Cornification to Iron Therapy}

\begin{tabular}{|c|c|c|c|c|c|c|c|c|c|c|c|}
\hline \multirow{2}{*}{$\begin{array}{l}\text { No. of } \\
\text { Cases }\end{array}$} & \multicolumn{10}{|c|}{ Haemoglobin (g.\%) } & \multirow{2}{*}{ Total } \\
\hline & $4 \cdot 0-4.9$ & $5 \cdot 0-5 \cdot 9$ & $6 \cdot 0-6 \cdot 9$ & $7 \cdot 0-7 \cdot 9$ & $8 \cdot 0-8 \cdot 9$ & $9 \cdot 0-9 \cdot 9$ & $10 \cdot 0-10.9$ & $11 \cdot 0-11 \cdot 9$ & $12 \cdot 0-12 \cdot 9$ & $13 \cdot 0-13.9$ & \\
\hline $\begin{array}{cc}\text { Group } & 1 \\
,, & \mathbf{2}\end{array}$ & $\frac{1}{1}$ & $\frac{7}{1}$ & $\begin{array}{l}2 \\
4 \\
3\end{array}$ & $\begin{array}{l}6 \\
2 \\
4\end{array}$ & $\begin{array}{l}7 \\
2 \\
4\end{array}$ & $\begin{array}{l}7 \\
3 \\
9\end{array}$ & $\begin{array}{l}3 \\
6 \\
2\end{array}$ & $\begin{array}{l}5 \\
1 \\
1\end{array}$ & $\begin{array}{r}1 \\
1 \\
\end{array}$ & $\frac{-}{1}$ & $\begin{array}{l}39 \\
20 \\
25\end{array}$ \\
\hline
\end{tabular}

It was possible to keep four of the patients in Group 2 under prolonged observation while they

TABLE

DEGREE OF ANAEMIA IN THREE GROUPS OF ANAEMIC PATIENTS UNDER INVESTIGATION 


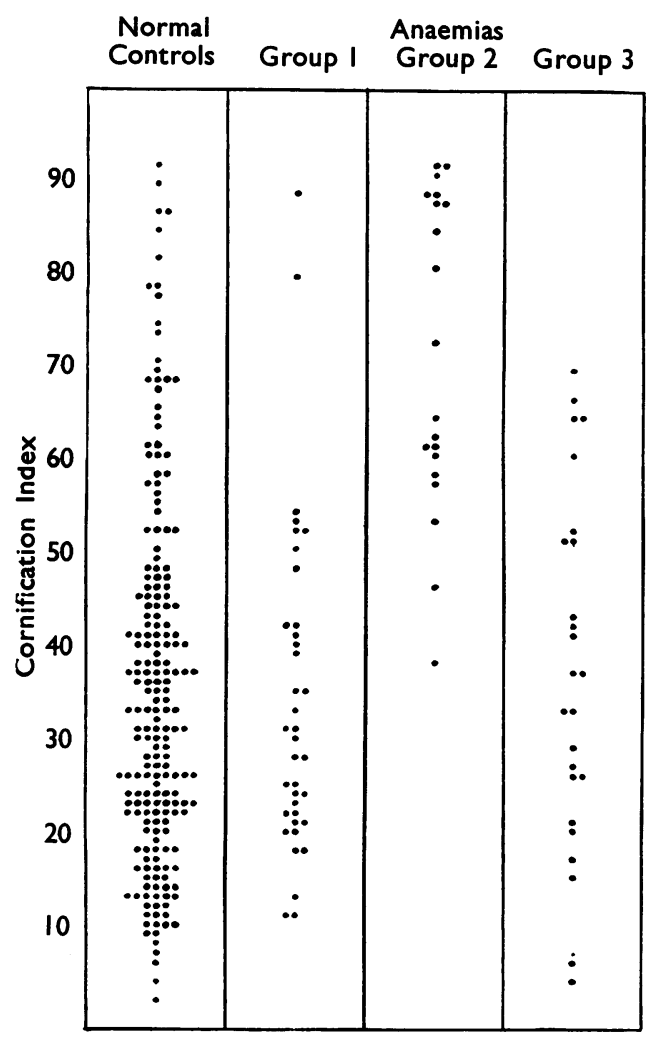

Miss N. W.

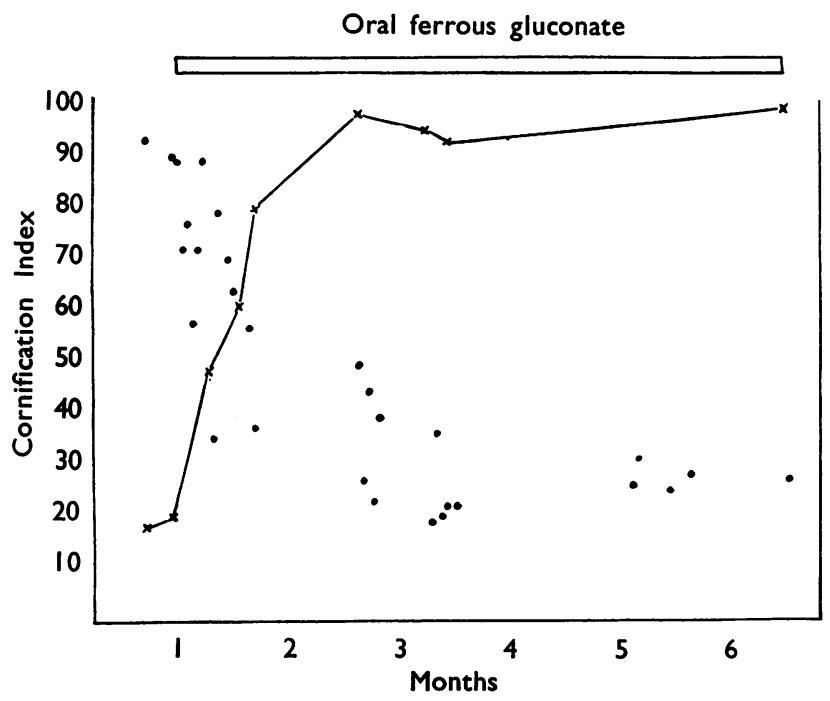

Fig. 2.-Response to oral iron therapy in Miss N. W. aged 31 years. $\mathbf{X} \times$ Haemoglobin. Cornification index.

FIG. 1.-Cornification indices of 200 normal subjects compared with three groups of anaemic patients.

Miss A. H.

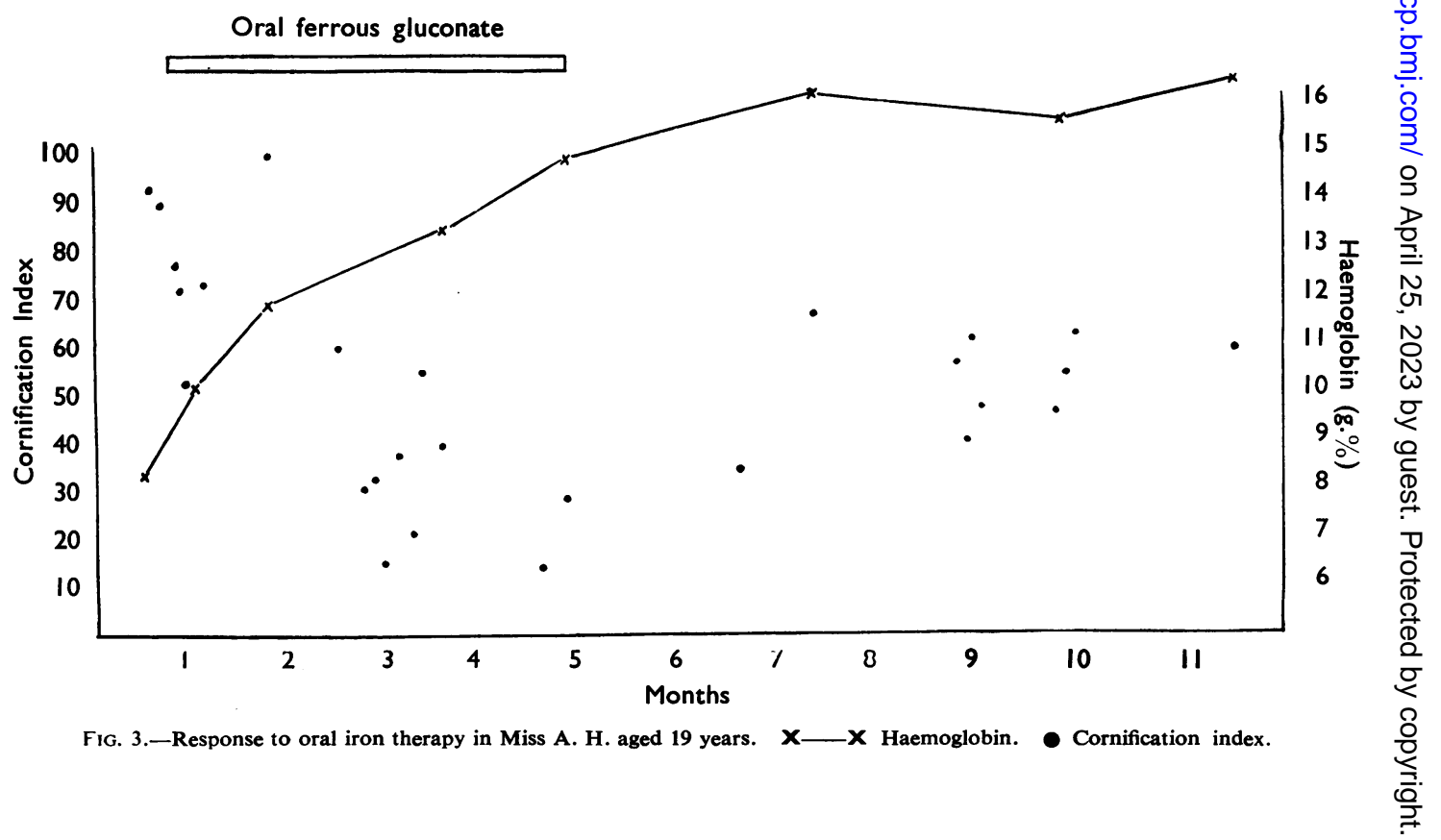


were treated with iron. In two cases treated with ferrous gluconate, 0.6 g. t.d.s. by mouth, haematological recovery was complete and at the same time the buccal cornification fell to within normal limits (Figs. 2 and 3). Miss A. H. discontinued treatment after four months and although her blood picture remains normal there seems to be a tendency towards increased buccal cornification again. One case treated with "imferon" and another treated with both "imferon" and ferrous gluconate showed similar changes in the buccal epithelium during treatment.

\section{Discussion}

There is no evidence that buccal cornification is affected by anaemia itself. The findings in patients with leukaemia and pernicious anaemia were not different from the normal although the degree of anaemia in these cases was as severe as in the iron-deficiency groups. In the patients with iron deficiency there is no correlation between increased cornification and the severity of the anaemia.

The two groups of patients with iron-deficiency anaemia form clinically distinct entities and it is possible that the abnormal buccal cornification in the second group is related to the different development of the disease. In the first group the anaemia is known to be due to blood loss alone, but the women with "idiopathic" iron-deficiency anaemia have no evidence of haemorrhage. Menorrhagia, although sometimes associated with this syndrome, is often not severe and may be corrected by treatment of the anaemia. Other factors, including dietary defects, constitutional gastric atrophy, malabsorption of iron, and excessive childbearing, have been invoked to explain the pathogenesis of this disorder. The essential feature of this "idiopathic" anaemia, whatever its primary cause, is its chronicity (Moore, 1950).

The epithelial changes noted above may be related to the tissue stores of iron rather than to the anaemia itself. In pernicious anaemia and the leukaemias there is no excess loss of iron from the body and the reserves may even be increased if blood transfusions have been given. In "idiopathic" hypochromic anaemia the disease usually pursues a chronic relapsing course-often over several decades. Patients with this disorder tolerate low haemoglobin levels for long periods before seeking medical treatment and then do so only because the severity of the anaemia makes normal life impossible. These patients have grossly depleted iron stores which probably cannot be fully replenished by oral iron therapy alone. Cases of gastro-intestinal haemorrhage do not commonly remain untreated long enough for the iron stores to become so grossly depleted. Where such cases do remain undetected for a long period the patient will probably present as a case of "idiopathic" anaemia with the associated oral changes.

Exhaustion of the body's supply of iron will lead to an intracellular deficiency and consequent disorder of cellular metabolism. Epithelial cornification seems to be affected by this disorder and the return of buccal cornification to normal under the influence of iron therapy indicates that iron is in some way concerned with the normal development of squamous epithelium.

Some cases showing a high cornification index were found in the healthy controls, but as they were not investigated haematologically it is quite possible that some subjects with overt or latent iron deficiency were included in this group.

\section{Summary}

The buccal cornification, as reflected in Papanicolaou-stained smears, has been estimated in patients with iron-deficiency and other anaemias.

Women with "idiopathic" hypochromic anaemia show an abnormal increase in buccal cornification which has been shown in some cases to revert to normal after iron therapy. This is not related to the degree of anaemia but may be due to decreased tissue iron stores.

I wish to thank Dr. S. Shaw for his encouragement in this work.

\section{REFERENCES}

Jacobs, A. (1959). Brit. dent. J., 106, 249.

Moore, C. V. (1950). Blood, 5 8,76.

Paterson, D. R. (1919). J. Laryng., 34, 289

Waldenström, J. (1938). Acta med. scand., Suppl. 90, p. 830. 